\section{$\underset{\substack{\text { hommes } \\ \text { \& migrations }}}{ }$}

\section{Hommes \& migrations}

Revue française de référence sur les dynamiques

migratoires

$1282 \mid 2009$

Santé et droits des étrangers : réalités et enjeux

\title{
Regards sur la Biennale africaine de la photographie 2009 à Bamako
}

A travers deux artistes programmés dans le in et le off

\section{Dagara Dakin}

\section{(2) OpenEdition}

Journals

Édition électronique

URL : http://journals.openedition.org/hommesmigrations/466

DOI : 10.4000/hommesmigrations.466

ISSN : 2262-3353

Éditeur

Musée national de l'histoire de l'immigration

Édition imprimée

Date de publication : 1 novembre 2009

Pagination : 157-163

ISSN : 1142-852X

Référence électronique

Dagara Dakin, «Regards sur la Biennale africaine de la photographie 2009 à Bamako », Hommes \& migrations [En ligne], 1282 | 2009, mis en ligne le 29 mai 2013, consulté le 22 septembre 2020. URL http://journals.openedition.org/hommesmigrations/466 ; DOI : https://doi.org/10.4000/

hommesmigrations. 466

Ce document a été généré automatiquement le 22 septembre 2020.

Tous droits réservés 


\section{Regards sur la Biennale africaine de la photographie 2009 à Bamako}

A travers deux artistes programmés dans le in et le off Dagara Dakin

\section{Frontières constrastées}

1 De ces regards multiples, il faut souligner le discours tout en retenue du Réunionnais Yo-Yo Gonthier. Son travail, qualifié par l'écrivain Manthia Diawara de "discret et intelligent", révèle comment, à l'île Maurice, des plages sont progressivement devenues des propriétés privées. Ces images disent, tout en subtilité, la violence sourde des frontières qui s'instaurent sur ces espaces de liberté entre riches et pauvres.

2 La frontière, c'est aussi celle qui exclut du fait des différences. Différences qui s'expriment par la couleur de la peau comme dans la série de portraits d'albinos du jeune prodige Seydou Camara, issu du Cadre de formation et de promotion en photographie. Le respect qu'il porte à ses sujets transparaît dans la justesse de son regard et la manière dont il les photographie.

3 La question de l'homosexualité, et le rejet qu'il induit dans les sociétés africaines, est abordée dans la vidéo du Nigérian Andrew Esiebo. Quant aux photographies de la SudAfricaine Zanele Muholi extraites de la série Miss D'vine, elles abordent la question de la transsexualité.

4 Si l'on savait que ces questions étaient objet de débat dans la société sud-africaine, le tabou dont elles sont souvent revêtues dans les autres pays africains semble interroger les artistes qui y vivent. Zanele Muholi a clairement affiché sa volonté de faire changer les mentalités sur ces questions lors du discours qu'elle a prononcé en recevant le prix Casa Africa. Ce prix, qui distingue une photographe résidant en Afrique, permet la publication d'une monographie, l'édition d'une collection spécialisée et une exposition monographique à la Palmas (Canaries), puis itinérante. 


\section{Espoirs déçus}

$5 \quad$ La série intitulée Espoir déchu des Darfouris au Caire de l'Égyptienne Myriam Abdelaziz nous rappelle que l'Europe n'est pas le seul Eldorado qui fait le désespoir de nombre de migrants. Ce reportage photographique porte sur l'exclusion dont font l'objet les Darfouris au Caire - lieu où ils espéraient trouver de meilleures conditions de vie.

De la même manière, la Sud-Africaine Jodi Bieber - lauréate du Prix de l'Union européenne, distinguant le meilleur photographe de presse ou de reportage - nous remet en mémoire la politique de reconduite à la frontière des clandestins que pratique l'Afrique du Sud. Elle nous rappelle que la gestion de la question des migrations illégales en Afrique présente des similitudes avec la politique migratoire européenne. On a tendance en effet à l'oublier, mais de plus en plus d'accords sont passés entre les pays du Sud et ceux du Nord. Mais au-delà, les politiques africaines ont tendance à calquer leur manière de faire sur le modèle occidental.

\section{Entre réel et fiction}

7 Face à ces reconduites à la frontière, le Sud-Africain Graeme Williams avance que "la seule mesure pour un changement durable en Afrique du Sud ne peut être qu'une réelle amélioration effective de la vie des pauvres". Sa réponse se traduit en photographie par des images très colorées où des personnages réels côtoient des figures dessinées, habituellement présentes dans l'espace public sous des formes diverses telles qu'images publicitaires, mannequins, etc. Il ne s'agit pas de mises en scène, mais d'instants captés par le regard exercé du photographe. Il joue ainsi des contrastes entre condition sociale de ses sujets et images de l'espace public. L'effet d'ensemble laisse à penser que l'artiste cherche à souligner la frontière existant entre vie rêvée et vie vécue de ses protagonistes.

8 Mohammed Bourrouissa, l'une des révélations de la scène photographique actuelle, joue également dans ses mises en scène des notions que sont le réel et la fiction. Son langage emprunte aux images que l'on retrouve dans nombre de quotidiens ou magazines et qui ont pour sujet les Périphéries (titre de la série exposée dans le musée), soit les banlieues françaises aussi appelés "quartiers populaires". L'artiste revisite ainsi ces "clichés", qu'il interroge. De la sorte, il oblige le spectateur à une attention plus accrue quant à la supposée vérité des images.

\section{Beach, une série photographique de Yo-Yo Gonthier}

9 Dans la salle de l'exposition internationale des Rencontres de Bamako, la série Beach du photographe réunionnais Yo-Yo Gonthier semblait, de prime abord, ne pas correspondre à la thématique des frontières, axe autour duquel s'articulait cette manifestation biennale. Pourtant, elle y répondait, mais pas de manière évidente, plutôt tout en discrétion.

10 Sur les cimaises du musée, le spectateur pouvait admirer six grandes photographies de format carré - le format de prédilection de l'artiste -, le tout disposé en carré décidément... La lecture de ces images se fait comme on lit une bande dessinée, soit vignette après vignette, image après image. En premier lieu, si le spectateur fait le 
choix d'une lecture classique de la gauche vers la droite, on voit une plage déserte mais attrayante. Puis, sur la seconde image un panneau indicateur signale la présence de caméras de surveillance sur la plage. Mais on n'y prête pas attention outre mesure. Simple signe plastique peut-être, pense-t-on sur l'instant. Ensuite vient une vue de la même plage, mais prise sous un autre angle. Et là, on s'aperçoit que des barrières bordent par endroit la plage et en interdisent l'accès. Le panorama suivant nous en dit un peu plus puisqu'il figure un poteau métallique au sommet duquel est juchée une caméra de surveillance. Le décor est planté et le propos se fait plus clair lorsque vient ce que le spectateur peut considérer comme la dernière image, à savoir la seule sur laquelle apparaît une figure humaine, mais vue de dos. Il s'agit d'un gardien en uniforme kaki portant casque colonial sur la tête et talkie-walkie à la ceinture. Plage sous surveillance donc.

La série comprend initialement un nombre plus important de photographies, mais, comme c'est bien souvent le cas dans les expositions de l'envergure des Rencontres, elle est morcelée. Toutefois, dans le cas présent, le propos de l'artiste, même s'il n'est vu que de façon partielle, reste lisible. Il y manque cependant toute une série de photographies sur lesquelles l'on aurait pu voir ce qui constitue en quelque sorte le pendant à cette privatisation progressive de ces espaces de liberté que sont censés être initialement les plages. Il s'agit de petites baraques de commerçants ambulants installées non loin de la plage. Elles illustrent, du point de vue de l'artiste, un contrepoint à ces zones privatisées. Le photographe parle, lui, de poches de résistance face à la domination capitaliste actuelle.

\section{Paradis perdu}

12 Ce qui fait la force de ces images, outre leur qualité plastique, c'est la manière dont elles abordent la question de la frontière. Mais il s'agit bien plus que de frontière, il y est question de ségrégation, de séparation, de marginalisation. Le photographe ne dénonce pas tant la violence des rapports entre riches et pauvres, il la pointe du doigt, en révèle le côté sournois, lent et progressif. Dans un premier temps, il semble nous inviter à la contemplation d'un paysage balnéaire, mais au final nous nous retrouvons face à des murs de protection érigés qui nous interdisent toute évasion. La violence qui sourd de ces images en est d'autant plus percutante. Il se dégage de l'ensemble une sensation de promesse non tenue, d'espoir déchu. En jouant des oppositions entre paysage, disons idyllique, et système sécuritaire, entre sentiment de liberté et barrière, voire enfermement, cloisonnement, l'artiste nous fait pleinement ressentir ce que peut signifier pour certains la privatisation de ces espaces publics que sont à l'origine les plages. Son discours est sans ambiguïté, mais il n'est pas livré de façon brute. Yo-Yo Gonthier n'impose pas son point de vue. Il attend du spectateur qu'il se fasse sa propre opinion. Il y va donc par petites touches discrètes et, progressivement, nous fait voir puis comprendre ce vers quoi il attire notre regard. Il est plus artiste du dévoilement que de la révélation. La vérité est un cheminement, semble nous suggérer la manière dont il procède. Certes, nous savons que ce discours est tronqué du fait que, sur la série, seules six photographies ont été sélectionnées. Ceci étant, le plasticien a beau avoir réalisé des images présentant ce qu'il considère comme des poches de résistance, il n'en demeure pas moins que le sentiment qui l'emporte est celui de la violence faite à ceux qui ne pourront plus accéder à ces espaces maintenant dédiés uniquement aux loisirs. 


\section{À force d'images}

13 qu'il s'impose et auxquelles il ne veut déroger. Cela transparait même dans le choix du format de ses images. Il fait de la photographie comme d'autres font de l'estampe, de la calligraphie ou écrivent des haïkus. Ce n'est pas un hasard si la vague de Hokusai est une de ses images de chevet. Ce n'est pas un hasard non plus si, bien souvent, à l'origine de certaines de ses photographies, il y a un dessin. Il veut aller à l'essentiel, ne pas se perdre en tergiversations. Mais dans le même temps, il ne se précipite pas. L'essentiel exige de l'attention, de la concentration. "Il ne faut pas confondre vitesse et précipitation", dit l'adage. Yo-Yo Gonthier ne veut pas qu'il y ait de confusion, que l'on se méprenne sur ce qu'il veut dire. Ce qui par moment peut être un handicap, car il y a toujours un moment où il faut dire. Mais c'est de cette tension et de cette dynamique qu'il tire la force de son propos, la force de ses images.

\section{(In) Secure, une vidéo de Dimitri Fagbohoun}

CEuvre du plasticien franco-béninois Dimitri Fagbohoun, la vidéo intitulée (In) Secure fut présentée pour la première fois lors du Festival panafricain d'Alger en juillet 2009. D'une durée de six minutes, elle aborde sous un angle particulier la question de l'immigration illégale, et plus précisément les conditions de vie qu'engendre ce type de migration.Le titre de la vidéo (In) Secure cherche à faire entendre dans une même locution un sens et son contraire. Soit, à la fois, la notion de sécurité et celle d'insécurité. Ce titre renvoie au contenu de la vidéo, lequel présente des portraits de vigiles chargés d'assurer la sécurité de lieux tels que des bâtiments officiels, des banques, des hôpitaux, etc. Mais ces vigiles ont la particularité d'être eux-mêmes en situation d'insécurité, parce que sans papiers et donc menacé de reconduite à la frontière. Ce que l'artiste veut pointer du doigt, c'est bien évidemment le paradoxe de la situation de ces vigiles, et plus particulièrement l'état psychologique dans lequel ils sont plongés du fait de leur condition d'illégaux. On peut se demander, en effet, comment dans leur situation ces personnes peuvent être les garantes d'une protection dont elles-mêmes ne bénéficient pas. Elles sont considérées comme illégales, par conséquent elles ne devraient pas occuper ces postes. Mais la réalité économique en fait des candidats idéaux pour ce type d'activité. Ils sont une main-d'œuvre bon marché, qui ne peut avoir d'exigence particulière, parce que fragilisée par son statut de hors-laloi.

\section{De la photographie d'identité}

Les photographies d'identité, qui se succèdent à l'écran, ont été réalisées en vue d'établir des badges de service. Elles n'ont pas été prises par l'artiste. Il les a récupérées à l'insu des protagonistes. L'artiste s'en sert comme d'un matériau, les faisant se succéder par le truchement de divers procédés, qui alternent fondu enchainé, images colorisées, images radiographiques ou comme obtenues à partir d'une vision en infrarouge. Ce qui renvoie à l'imagerie sécuritaire. Cela étant, ces photographies ont beau être des photographies dites "d'identité", elles ne nous renseignent en rien sur 
l'identité des protagonistes. Ce que nous appelons communément "photographie d'identité" ne suffit pas à établir l'identité d'une personne. Elle doit s'accompagner d'un certain nombre d'éléments pour prendre sens. Les portraits qui se succèdent à l'écran ont beau être des photographies au format des photographies d'identité, ces personnes demeurent des anonymes aux yeux du spectateur.

Le propos de l'artiste n'est pas de mettre un nom sur ces portraits, mais plutôt de donner un visage à un état ou un phénomène d'ordre psychologique. Comme il le dit lui-même :

"Au-delà de toute critique sur leur condition sociale, c'est l'aspect humain, psychologique qui m'intéressait. En effet, il y a une espèce de schizophrénie à aller travailler pour une entreprise de sécurité alors que l'on peut être arrêté à tout moment comme un hors-la-loi. C'est ce sentiment que je cherche à retranscrire."

\section{Le dispositif sonore}

17 Si la vidéo est une affaire d'images, c'est aussi une affaire de sons et, dans le cas présent, les unes ne fonctionnent pas sans les autres. Le dispositif sonore se présente comme un assemblage hétéroclite de sons. Des pulsations cardiaques rythment la succession des images. Puis viennent des bruits de bottes qui font penser à une marche militaire. L'extrait d'un discours du leader noir américain, Malcom X, vient interférer, comme un court-circuit historique, tandis que, progressivement, en arrière-plan de ces sons, des voix se font entendre. Dans un premier temps, elles font penser à une scène qui se déroulerait dans un bar ou un quelconque lieu très fréquenté. Mais lorsqu'on comprend ou que l'on apprend qu'il s'agit de l'enregistrement d'une scène de reconduite à la frontière enregistrée dans un aéroport espagnol, le tout prend une autre dimension.

Dès lors, le fond sonore se présente comme une menace en toile de fond, un peu comme une épée de Damoclès qui serait suspendue au-dessus de la tête de ces personnes. Le rythme cardiaque diffuse un sentiment d'oppression, l'angoisse est palpable. Quant à la marche militaire, elle vient comme pour appuyer et renforcer toutes ces sensations. Dimitri Fagbohoun essaie, par le biais des matériaux que sont l'image et le son, de nous faire percevoir quelque chose qui est de l'ordre de l'affect, à savoir le sentiment d'insécurité. Un sentiment que nous connaissons tous à des degrés divers, mais qui est, semble-t-il, le quotidien de ces anonymes dont il tente ici d'esquisser un portrait psychologique. L'artiste ne prend pas de distance avec le sujet. Il en sonde plutôt les affects, prenant par la même occasion le risque de tomber dans le pathos. Toutefois, on ne saisit pas pleinement la mesure du propos si on en reste là. Le titre de l'œuvre a son importance, qui nous ouvre une lecture plus politiquement marquée. Il y est question de sécurité et d'insécurité, deux termes que le discours politique emploie régulièrement quand il est question d'immigration ou d'une certaine catégorie d'étranger, pour être plus exact. Si ce thème n'est pas clairement énoncé, il revient malgré tout de façon, disons, sous-entendu.

Le double sens du titre (In) Secure a son importance puisqu'il souligne la dimension toute relative de la notion de sécurité. Il ne s'agit pas d'une coquetterie de la part de l'artiste. En effet, le sentiment d'insécurité engendre la quête de la sécurité. Seulement, le sentiment que procure la sécurité ne se préserve qu'au moyen de toute une série de dispositifs que connaît bien notre époque et qui, tel un puits sans fond, tire 
graduellement vers la surenchère sécuritaire. Par le double sens que renferme le titre de sa vidéo, Dimitri Fagbohoun souligne les effets pervers et les contradictions que renferme le discours sécuritaire. Lequel discours, bien souvent, accompagne la question migratoire dans la bouche des politiques.

La multiplicité des points de vue, de même que la variété des sujets présentés dans cette exposition, illustre parfaitement la complexité de la question des frontières. En parcourant de manière non exhaustive l'exposition internationale - présentée au musée national du Mali - il était important de voir la richesse de la production photographique sur le continent, mais aussi au-delà. On sort de cette exposition en ayant éprouvé la complexité des interrogations que pose la notion de frontière à notre époque dite "de globalisation".

\section{ANNEXES}

\section{Biographie}

Yo-Yo Gonthier est né à Niamey, au Niger, en 1974. Photographe plasticien indépendant, il questionne actuellement l'effacement de la mémoire dans une société occidentale où la vitesse, le progrès et la technologie semblent être les valeurs essentielles. Sa démarche plastique s'articule autour du surgissement du merveilleux à travers une interprétation particulière de la nuit et du clair-obscur. Son travail d'investigation nocturne a déjà fait l'objet de plusieurs parutions, et notamment dans l'ouvrage intitulé Les lanternes sourdes en 2004. Par ailleurs, il s'intéresse aux vestiges de l'Empire colonial français et aux frottements entre Histoire et mémoires. Une première étape du projet Outre-mer a été présentée à l'espace Khiasma, aux Lilas, en juin 2008. Il a présenté un travail de commande sur le monde créole dans le cadre de l'exposition Kréyol Factory en avril 2009 au Parc de la Villette. Sélectionné dans la section internationale lors de la dernière édition 2009 des Rencontres africaines de la photographie de Bamako, il y a exposé une partie de sa série intitulée "Beach".

Dimitri Fagbohoun est né en 1972 d'un père béninois et d'une mère ukrainienne. Il utilise la photographie, la vidéo et l'installation comme moyens d'expression. Dans son œuvre, il s'intéresse aux questions ayant trait à l'identité, la politique et la mémoire. Il a notamment exposé dans le cadre des Rencontres africaines de la photographie de Bamako en 2007.

\section{RÉSUMÉS}

L'édition 2009 des Rencontres photographiques de Bamako, qui s'est tenue du 7 novembre au 7 décembre, renouvelle son pari d'ouverture des frontières du monde et du marché de l'art en particulier. Cette huitième édition se consacre justement aux Frontières - géopolitiques, sociales, 
culturelles ou tout simplement humaines. Les réponses des artistes ont révélé non seulement l'actualité, la complexité, mais aussi le caractère contrasté des points de vue sur la question. En somme, le pari est déjà gagné quand les différences peuvent aussi réunir.

\section{AUTEUR}

DAGARA DAKIN

Doctorant en histoire de l'art à l'université de Paris I 